\title{
ON SOME THEOREMS OF LITTLEWOOD AND SELBERG III
}

\author{
K. Ramachandra and A. Sankaranarayanan
}

\section{Introduction}

In paper II with the same title [2] we proved some unconditional results about $\zeta(s)$ in a more general set up. In this paper we continue these investigations. As before we begin by stating the final result of this paper as follows.

Theorem 1. Let $s=\sigma+i t$ and

$$
F(s)=\sum_{n=1}^{\infty} \frac{a_{n}}{n^{s}}=\prod_{p}\left(1-\frac{\omega(p)}{p^{s}}\right)^{-1}
$$

where $p$ runs over all primes and $\omega(p)$ are arbitrary complex numbers (independent of $s$ ) with absolute value not exceeding 1. Suppose $\alpha$ and $\delta$ are positive constants satisfying $\frac{1}{2} \leq \alpha \leq 1-\delta$ and that in $\{\sigma \geq \alpha-\delta, T-H \leq t \leq T+H\}$, $F(s)$ can be continued analytically and there $|F(s)|<T^{A}$. Here $A$ is a positive constant, $T \geq T_{0}, H=C \log \log \log T$ where $T_{0}$ and $C$ are large positive constants. Let $F(s) \neq 0$ in $\{\sigma>\alpha, T-H \leq t \leq T+H\}$. Then for $\alpha \leq \sigma \leq \alpha+C_{1}(\log \log T)^{-1}$, and $T-\frac{1}{2} H \leq t \leq T+\frac{1}{2} H$, we have,

(a) $\log |F(\sigma+i t)|$ lies between $C_{2}(\log T)(\log \log T)^{-1}$ and

$-C_{3}(\log T)(\log \log T)^{-1} \log \left\{C_{4}((\sigma-\alpha) \log \log T)^{-1}\right\}$ and

(b) $|\arg F(\sigma+i t)| \leq C_{5}(\log T)(\log \log T)^{-1}$,

where $C_{1}, C_{2}, C_{3}, C_{4}$ and $C_{5}$ are certain positive constants.

Corollary 1. For $\alpha+C_{1}(\log \log T)^{-1} \leq \sigma \leq 1-\delta, t=T$, we have,

$$
|\log F(\sigma+i t)| \leq C_{6}(\log T)^{\theta}(\log \log T)^{-1}
$$

where $\theta=(1-\sigma) /(1-\alpha)$ and $C_{6}$ is a positive constant.

Corollary 2. For $\alpha \leq \sigma \leq 1-\delta, t=T$, we have,

$$
|F(\sigma+i t)| \leq \exp \left(C_{7}(\log T)^{\theta}(\log \log T)^{-1}\right),
$$

where $\theta$ is as before and $C_{7}$ is a positive constant. 
Remark 1. The application of Theorem 1 to $\zeta(s)$ is clear by density results. Under the conditions of the theorem we can also prove density theorems for $F(s)$.

Remark 2. We now indicate the proof of the corollaries. Corollary 1 is already proved in [2] for the $\sigma$-range $\alpha+\delta \leq \sigma \leq 1-\delta$. The theorem above gives an upper bound $|\log F(\sigma+i t)| \leq C_{8}(\log T)(\log \log T)^{-1}$ for $\sigma=\sigma_{1}=$ $\alpha+(\log \log T)^{-1}$ and $T-H / 3 \leq t \leq T+H / 3$ (where $C_{8}>0$ is a constant). We have already an upper bound $|\log F(\sigma+i t)| \leq C_{8}(\log T)^{\theta}(\log \log T)^{-1}$ for $\sigma=\sigma_{2}=1-\delta$ and the same $t$-range. We now apply maximum modulus principle to the function (for suitable $X>0$ )

$$
\varphi(w)=(\log F(s+w)) X^{w} \exp \left(\left(\sin \frac{w}{100}\right)^{2}\right) .
$$

According to this its absolute value at $w=0$, namely $|\log F(s)|$ is majorised by its maximum modulus on the boundary of the rectangle $\left\{\sigma_{1}-\sigma \leq \operatorname{Re} w \leq \sigma_{2}-\right.$ $\sigma,-H / 10 \leq \operatorname{Im} w \leq H / 10\}$. Corollary 1 follows by a proper choice of $X$ as a suitable power of $\log T$. (The bound for $|\log F(s+w)|$ namely $O\left((\log T)^{20}\right)$, needed on the horizontal sides of the rectangle can be obtained by Borel-Carathéodory theorem). This completes the proof of Corollary 1. Corollary 2 follows from Corollary 1 and the part (a) of the theorem.

By a modification of our proof of Theorem 1 we can prove

Theorem 2. Let $1-\delta \leq \alpha \leq 1-10(\log \log T)^{-1}$. Then for $\alpha \leq \sigma \leq$ $\alpha+(\log \log T)^{-1}$ (in place of $\left.\left(\alpha \leq \sigma \leq \alpha+C_{1}(\log \log T)^{-1}\right)\right)$ the assertions (a) and (b) hold, provided $F(s) \neq 0$ in the region mentioned in Theorem 1.

From Theorems 1 and 2 we can prove by the methods of [3] theorems like

Theorem 3. Let $\lambda_{0}(>7 / 12)$ be a constant. Then for any fixed $k$, the number of lattice points $\left(n_{1}, n_{2}, \ldots, n_{k}\right)$ in the first quadrant of the $k$ dimensional Euclidean space such that

$$
X \leq n_{1} \cdots n_{k} \leq X+X^{\lambda_{0}}
$$

is given by

$$
X^{\lambda_{0}}(\log X)^{k-1}+O\left(X^{\lambda_{0}}(\log X)^{k-2}\right) .
$$

Remark. In fact we can prove an asymptotic formula valid uniformly for $k \leq \varepsilon(\log \log X)(\log \log \log X)^{-1}$. These and similar results will form the subject matter of another paper. Theorem 3 is due to M.N. Huxley and C. Hooley (unpublished).

In what follows we will prove only Theorem 1 . 


\section{Notation}

We use $z=x+i y, w=u+i v$ and $s=\sigma+i t$ in various contexts and we hope that this does not cause confusion. For any analytic function $F(s)$ we write $\left(F^{\prime} / F\right)(s)$ for $F^{\prime}(s) / F(s)$. The symbol $\equiv$ denotes a definition.

\section{Proof of Theorem 1}

It suffices to prove the theorem for $t=T$. Because we can consider a larger $H$, and every point of the smaller interval $T-\frac{1}{2} H \leq t \leq T+\frac{1}{2} H$ will be a mid point $\tau$ of a bigger interval of the type $\tau-\frac{1}{2} H \leq t \leq \tau+\frac{1}{2} H$ contained in $[T-H, T+H]$. We split the proof into three parts. The first two parts deal with an upper bound for a positive quantity $J_{0}$ in the form $O\left((\log T)(\log \log T)^{-1}\right)$. The third part deals with an application of this result to the proof of the theorem.

Lemma 1. Let $z=x+i y$ be a complex variable and

$$
F(z)=\sum_{n=1}^{\infty} a_{n} n^{-z}=\prod_{p}\left(1-\omega(p) p^{-z}\right)^{-1}
$$

where $p$ runs over all the primes and $\omega(p)$ are complex numbers independent of $z$ with $|\omega(p)| \leq 1$. Let $F(z)$ be regular in $\{x \geq \alpha-\delta, T-H \leq y \leq T+H\}$ and there $|F(z)|<T^{A}$ where $A>0$ is a constant. Here $T \geq T_{0}, \frac{1}{2} \leq \alpha \leq 1-\delta$, $H=C \log \log \log T$ where $\delta$ is a small positive constant, $\alpha$ is a constant and $T_{0}$ and $C$ are large positive constants. Put $z_{0}=2+i y$. Then for $z_{1}=x_{0}+i y_{0}$ where $T-\frac{1}{2} H \leq y_{0} \leq T+\frac{1}{2} H$ with $\alpha-\delta_{1} \leq x_{0} \leq 2$, we have

$$
\left|\frac{F^{\prime}}{F}\left(z_{1}\right)-\sum_{\rho \in D} \frac{1}{z_{1}-\rho}\right| \ll \log T
$$

where $\rho$ runs over all the zeros of $F(z)$ in the disc $D=D\left(z_{0}, 2-\alpha+2 \delta_{1}\right)$ defined by

$$
\left|z-z_{0}\right| \leq 2-\alpha+2 \delta_{1} .
$$

Here $\delta_{1}$ is any positive constant. We will suppose $11 \delta_{1}<\delta$.

Proof. This is Lemma 3 of [2].

Lemma 2. Under the conditions of Lemma 1, we have,

$$
\operatorname{Re} \frac{F^{\prime}}{F}\left(x_{0}+i y_{0}\right)=\sum_{\rho \in D} \frac{x_{0}-\beta}{\left(x_{0}-\beta\right)^{2}+\left(y_{0}-\gamma\right)^{2}}+O(\log T)
$$

where we have written $\rho=\beta+i \gamma$. This holds in particular for $\alpha \leq x_{0} \leq 2$. 


\section{Another expression for the left-hand side of (5)}

Lemma 3. Let $s=\sigma+i$ where $\alpha+(\log X)^{-1}=\sigma_{1} \leq \sigma \leq 1-\delta$ and $X=(\log T)^{\lambda}$ with some positive constant $\lambda<1$. Let $B(\geq 100000)$ be a constant. Then

$$
\begin{aligned}
I & \equiv \frac{1}{2 \pi i} \int_{u=2} \frac{F^{\prime}}{F}(s+w)\left(\frac{X^{2 w}-X^{w}}{w^{2} \log X}\right) \exp \left(\left(\sin \frac{w}{B}\right)^{2}\right) d w \\
& =O\left((\log T)^{\lambda}(\log \log T)^{-1}\right) .
\end{aligned}
$$

Proof. We have,

$$
\frac{1}{2 \pi i} \int_{u=2}\left(\frac{X}{n}\right)^{w} \exp \left(\left(\sin \frac{w}{B}\right)^{2}\right) \frac{d w}{w^{2} \log X}=(\log X)^{-1} \log \frac{X}{n}+O\left(\frac{n}{X \log X}\right),
$$

if $n \leq X$ and

$$
\frac{1}{2 \pi i} \int_{u=2}\left(\frac{X}{n}\right)^{w} \exp \left(\left(\sin \frac{w}{B}\right)^{2}\right) \frac{d w}{w^{2} \log X}=O\left(\frac{X}{n \log X}\right)
$$

if $n \geq X$.

Hence for any constant $\varepsilon(0<\varepsilon<1)$, we have,

$$
\frac{1}{2 \pi i} \int_{u=2}\left(\left(\frac{X^{2}}{n}\right)^{w}-\left(\frac{X}{n}\right)^{w}\right) \exp \left(\left(\sin \frac{w}{B}\right)^{2}\right) \frac{d w}{w^{2} \log X}=1+O\left(\frac{n}{X \log X}\right)
$$

if $n \leq X$ and

$\frac{1}{2 \pi i} \int_{u=2}\left(\left(\frac{X^{2}}{n}\right)^{w}-\left(\frac{X}{n}\right)^{w}\right) \exp \left(\left(\sin \frac{w}{B}\right)^{2}\right) \frac{d w}{w^{2} \log X}=O_{\varepsilon}\left(\left(\frac{X^{2}}{n}\right)^{\varepsilon}\left(\log X^{-1}\right)\right)$, if $X \leq n \leq X^{2}$ and

$$
\frac{1}{2 \pi i} \int_{u=2}\left(\left(\frac{X^{2}}{n}\right)^{w}-\left(\frac{X}{n}\right)^{w}\right) \exp \left(\left(\sin \frac{w}{B}\right)^{2}\right) \frac{d w}{w^{2} \log X}=O\left(\frac{X^{2}}{n \log X}\right)
$$

if $n \geq X^{2}$.

Thus if $1-\delta+\varepsilon<1$ we obtain

$$
\begin{aligned}
I & =O\left(\sum_{n \leq X} \frac{\Lambda(n)}{n^{\sigma}}+\sum_{X \leq n \leq X^{2}}\left(\frac{X^{2}}{n}\right)^{\varepsilon} \frac{\Lambda(n)}{n^{\sigma} \log X}+\sum_{n \geq X^{2}} \frac{X^{2} \Lambda(n)}{n^{1+\sigma} \log X}\right) \\
& =O\left(X^{2-2 \sigma}(\log X)^{-1}\right),
\end{aligned}
$$

where we have used $\Lambda(n)=\log p$ if $n=p^{m}, 0$ otherwise. This proves the lemma since $2 \lambda(1-\sigma) \leq \lambda$. 
Lemma 4. Let $3 V$ be asymptotic to $H$ and $|v| \leq V^{\prime}$ ( $V^{\prime}$ will be chosen to be asymptotic to $V$ ). Then for (fixed $s=\sigma+i t$ and all $w=u+i v$ ), $u+\sigma \geq \alpha-\delta_{1}$, we have,

$$
\left|\frac{F^{\prime}}{F}(s+w)-\sum_{\rho} \frac{1}{s+w-\rho}\right| \ll \log T
$$

where $\rho$ runs over all the zeros of $F(z)$ in the disc $D=D\left(z_{0}, 2-\alpha+2 \delta_{1}\right)$ defined by $\left|z-z_{0}\right| \leq 2-\alpha+2 \delta_{1}$ where $z_{0}=2+i t+i v$.

Proof. The proof follows from Lemma 1.

Lemma 5. Let

$$
\mu(\rho)=\frac{2^{s+w-\rho}-1}{(s+w-\rho)^{2} \log 2}
$$

and

$$
\mu=\sum_{\rho} \mu(\rho)
$$

where $\rho$ runs over all the zeros of $F(z)$ in "the rectangle" $R$ defined by

$$
R:\left\{\operatorname{Re} z \geq \alpha-2 \delta_{1},|t-y| \leq 2 V\right\} .
$$

Then for $|v| \leq V^{\prime}$ ( $V^{\prime}$ will be chosen asymptotic to $V$ ) and $u+\sigma \geq \alpha-\delta_{1}$, we have,

$$
\left|\frac{F^{\prime}}{F}(s+w)-\mu\right| \ll \log T .
$$

Proof. This is Lemma 6 of [2].

Lemma 6. It is possible to choose $V^{\prime}$ (asymptotic to $V$ ) such that on $v= \pm V^{\prime}$ and $u+\sigma \geq \alpha-10 \delta_{1}$, we have,

$$
\left|\sum_{\rho \in R} \mu(\rho)\right| \ll(\log T)^{2} .
$$

Proof. This is Lemma 7 of [2].

Remark. From now on we assume that $F(z) \neq 0$ in $\{x>\alpha, T-H \leq y \leq$ $T+H\}$. 
Lemma 7. We have,

$$
I=\frac{F^{\prime}}{F}(s)+I_{1}+I_{2}+S+O\left((\log T)^{\lambda}(\log \log T)^{-1}\right),
$$

where

$$
I_{2}=\frac{1}{2 \pi i} \int_{u=\alpha-\sigma-10 \delta_{1}} \mu\left(\frac{X^{2 w}-X^{w}}{w^{2} \log X}\right) \exp \left(\left(\sin \frac{w}{B}\right)^{2}\right) d w
$$

and

$$
S=\sum_{\rho \in R}\left(\frac{X^{2(\rho-s)}-X^{\rho-s}}{(\rho-s)^{2} \log X}\right) \exp \left(\left(\sin \left(\frac{\rho-s}{B}\right)\right)^{2}\right) .
$$

The two integrals in (14) are subject to $|v| \leq V^{\prime}$.

Proof. The proof follows by Cauchy's theorem of residues.

Lemma 8. For $\alpha+(\log X)^{-1}=\sigma_{1} \leq \sigma \leq 1-\delta$, we have,

$$
\begin{aligned}
& I_{1}=O\left(\left(X^{2 \alpha-2 \sigma-\delta_{1}}+X^{\alpha-\sigma-\delta_{1} / 2}\right) \frac{\log T}{\log \log T}\right), \\
& I_{2}=O\left(\left(X^{2 \alpha-2 \sigma-2 \delta_{1}}+X^{\alpha-\sigma-10 \delta_{1}}\right) \frac{\log T}{\log \log T}\right)
\end{aligned}
$$

and

$$
S=\sum_{\rho \in D} \frac{X^{2 \rho-2 s}-X^{\rho-s}}{(\rho-s)^{2} \log X}+O\left(\frac{\log T}{\log \log T}\right) .
$$

Proof. The estimates (15) and (16) follow from (11) and the fact that on $u=\alpha-\sigma-10 \delta_{1}$ we have $\mu=O(\log T)$ since $|s+w-\rho| \geq \delta_{1}$ on this line.

Lemma 9. For $\alpha+(\log X)^{-1}=\sigma_{1} \leq \sigma \leq 1-\delta$, we have,

$$
I_{1}=O\left(\frac{\log T}{\log \log T}\right), \quad I_{2}=O\left(\frac{\log T}{\log \log T}\right)
$$

and

$$
S_{0}=\omega \sum_{\rho \in D} \frac{X^{2 \beta-2 \sigma}+X^{\beta-\sigma}}{(\log X)\left((\sigma-\beta)^{2}+(t-\gamma)^{2}\right)}
$$

where $S_{0}$ denotes the sum in (17) and $\omega$ is a complex number (depending on other parameters) with $|\omega| \leq 1$. 
Proof. The proof follows from Lemma 8.

Lemma 10. For $\alpha+(\log X)^{-1}=\sigma_{1} \leq \sigma \leq 1-\delta$, we have,

$$
\begin{aligned}
& \sum_{\rho \in D} \frac{\sigma-\beta}{(\sigma-\beta)^{2}+(t-\gamma)^{2}} \\
& \quad=\omega^{\prime} \sum_{\rho \in D} \frac{\left(X^{2 \beta-2 \sigma}+X^{\beta-\sigma}\right)\left(\sigma_{1}-\alpha\right)}{(\sigma-\beta)^{2}+(t-\gamma)^{2}}+O(\log T)
\end{aligned}
$$

where $\omega^{\prime}$ is real and $\left|\omega^{\prime}\right| \leq 1$.

Proof. The proof follows from Lemmas 2, 3, 7, 8 and 9.

Lemma 11. We have,

$$
J_{0} \equiv \sum_{\rho \in D} \frac{\sigma_{1}-\beta}{\left(\sigma_{1}-\beta\right)^{2}+(t-\gamma)^{2}}=O(\log T) .
$$

Proof. Put $\sigma=\sigma_{1}$ in (20). We have $\beta-\sigma_{1} \leq \alpha-\sigma_{1}=-(\log X)^{-1}$ and also $\sigma_{1}-\alpha \leq \sigma_{1}-\beta$ and so

$$
J_{0}=\sum_{\rho \in D} \frac{\sigma_{1}-\beta}{\left(\sigma_{1}-\beta\right)^{2}+(t-\gamma)^{2}}=\omega^{\prime \prime}\left(\frac{1}{e^{2}}+\frac{1}{e}\right) J_{0}+O(\log T),
$$

where $\left|\omega^{\prime \prime}\right| \leq 1$

This proves the lemma since $e^{-2}+e^{-1}<1$ and $\left|\omega^{\prime \prime}\right| \leq 1$.

Lemma 12. For $S_{0}$ defined by (19) we have,

$$
S_{0}=w_{1} \sum_{\rho \in D}\left(\frac{X^{2 \beta-2 \sigma}+X^{\beta-\sigma}}{\left(\sigma_{1}-\beta\right)^{2}+(t-\gamma)^{2}}\right)\left(\sigma_{1}-\beta\right),
$$

with $\left|\omega_{1}\right| \leq 1$.

Proof. The lemma follows from $(\log X)^{-1}=\sigma_{1}-\alpha \leq \sigma_{1}-\beta$ and also $\sigma \geq \sigma_{1}$.

Lemma 13. With $\sigma_{1}=\alpha+(\log X)^{-1}$, we have,

$$
\log F\left(\sigma_{1}+i t\right)=O\left(\frac{\log T}{\log \log T}\right) .
$$

Proof. The lemma follows from

$$
\int_{\sigma_{1}}^{1-\delta_{1}} \frac{F^{\prime}}{F}(\sigma+i t) d t=O\left(\frac{\log T}{\log \log T}\right)-\log F\left(\sigma_{1}+i t\right)
$$

and the fact that here the left-hand side is (by Lemmas $2,3,7,8,9,11$ and 12) $O\left((\log T)(\log \log T)^{-1}\right)$. 
Lemma 14. For $\alpha \leq \sigma \leq \sigma_{1}$, we have,

$$
\left(\sigma_{1}-\sigma\right) \frac{F^{\prime}}{F}\left(\sigma_{1}+i t\right)=O\left(\frac{\log T}{\log \log T}\right) .
$$

Proof. The proof follows from Lemmas 2, 3, 7, 8, 9, 11 and 12.

Lemma 15. For $\alpha \leq \sigma \leq \sigma_{1}$ the quantity

$$
\int_{\sigma}^{\sigma_{1}} \operatorname{Re}\left(\frac{F^{\prime}}{F}\left(\sigma_{1}+i t\right)-\frac{F^{\prime}}{F}(u+i t)\right) d u
$$

does not exceed $C_{9}(\log T)(\log \log T)^{-1}$, but exceeds

$$
C_{10}(\log T)(\log \log T)^{-1}-C_{11}(\log T)(\log \log T)^{-1} \log \left(\left(C_{12}(\log \log T)(\sigma-\alpha)\right)^{-1}\right),
$$

where $C_{9}, C_{10}, C_{11}$ and $C_{12}$ are positive constants.

Proof. By Lemma 2 the quantity in question is

$$
\int_{\sigma}^{\sigma_{1}}\left(J\left(\sigma_{1}\right)-J(u)\right) d u+O\left(\frac{\log T}{\log \log T}\right)
$$

where

$$
J(u) \equiv \sum_{\rho \in D} \frac{u-\beta}{(u-\beta)^{2}+(t-\gamma)^{2}} .
$$

Now

$$
J\left(\sigma_{1}\right)-J(u)=\sum_{\rho \in D} \frac{\left(\sigma_{1}-u\right)(t-\gamma)^{2}}{Y}+\sum_{\rho \in D} \frac{\left(\sigma_{1}-\beta\right)(u-\beta)\left(u-\sigma_{1}\right)}{Y}
$$

where $Y=\left((u-\beta)^{2}+(t-\gamma)^{2}\right)\left(\left(\sigma_{1}-\beta\right)^{2}+(t-\gamma)^{2}\right)$. Denote the two sums in $J\left(\sigma_{1}\right)-J(u)$ by $\sum_{1}$ and $\sum_{2}$. We have

$$
\sum_{1} \leq \sum_{\rho \in D} \frac{\sigma_{1}-u}{\left(\sigma_{1}-\beta\right)^{2}+(t-\gamma)^{2}}
$$

and so

$$
\begin{aligned}
\int_{\sigma}^{\sigma_{1}} \sum_{1} d u & \leq \sum_{\rho \in D} \frac{\frac{1}{2}\left(\sigma_{1}-\sigma\right)^{2}}{\left(\sigma_{1}-\beta\right)^{2}+(t-\gamma)^{2}} \\
& \leq \frac{1}{2}(\log X)^{-1} \sum_{\rho \in D} \frac{\sigma_{1}-\beta}{\left(\sigma_{1}-\beta\right)^{2}+(t-\gamma)^{2}} \\
& =\frac{1}{2} J_{0}(\log X)^{-1}=O\left((\log T)(\log \log T)^{-1}\right)
\end{aligned}
$$


Now $\sum_{2}$ is negative and

$$
\begin{aligned}
-\int_{\sigma}^{\sigma_{1}} \sum_{2} d u & =\int_{\sigma}^{\sigma_{1}} \sum_{\rho \in D} \frac{(u-\beta)\left(\sigma_{1}-\beta\right)\left(\sigma_{1}-u\right)}{Y} d u \\
& \leq \int_{\sigma}^{\sigma_{1}} \sum_{\rho \in D}\left(\frac{\sigma_{1}-u}{u-\alpha} \cdot \frac{(u-\beta)^{2}\left(\sigma_{1}-\beta\right)}{Y}\right) d u \\
& \leq J_{0}(\log X)^{-1} \int_{\sigma}^{\sigma_{1}} \frac{d u}{u-\alpha}=O\left(\frac{\log T}{\log \log T} \log \frac{\sigma_{1}-\alpha}{\sigma-\alpha}\right) .
\end{aligned}
$$

This proves the lemma.

Lemma 16. For $\alpha \leq \sigma \leq \sigma_{1}$, we have,

$$
\int_{\sigma}^{\sigma_{1}} \operatorname{Im}\left\{\frac{F^{\prime}}{F}\left(\sigma_{1}+i t\right)-\frac{F^{\prime}}{F}(u+i t)\right\} d u=O\left(\frac{\log T}{\log \log T}\right) .
$$

Proof. For $\alpha \leq \sigma \leq \sigma_{1}$ we see that the integrand is (apart from a term of the type $O(\log T))$, by Lemma 2 ,

$$
\begin{gathered}
\sum_{\rho \in D}\left(\frac{-(t-\gamma)}{\left(\sigma_{1}-\beta\right)^{2}+(t-\gamma)^{2}}-\frac{-(t-\gamma)}{(u-\beta)^{2}+(t-\gamma)^{2}}\right) \\
=\sum_{\rho \in D} \frac{(t-\gamma)\left(\left(\sigma_{1}-\beta\right)^{2}-(u-\beta)^{2}\right)}{Y}
\end{gathered}
$$

and hence its absolute value is

$$
\leq \sum_{\rho \in D}|t-\gamma|\left(\sigma_{1}-u\right)\left(2 \sigma_{1}-2 \beta\right) Y^{-1} .
$$

Hence the absolute value of the integral in question is

$$
\begin{aligned}
& \leq \frac{1}{\log X} \sum_{\rho \in D}\left\{\left(\int_{\sigma}^{\sigma_{1}} \frac{|t-\gamma|}{(u-\beta)^{2}+(t-\gamma)^{2}} d u\right) .\right. \\
& \left.\cdot\left(\frac{2\left(\sigma_{1}-\beta\right)}{\left(\sigma_{1}-\beta\right)^{2}+(t-\gamma)^{2}}\right)\right\} \\
& \leq \frac{2}{\log X} \sum_{\rho \in D}\left\{\left(\int_{\beta}^{\infty} \frac{|t-\gamma|}{(u-\beta)^{2}+(t-\gamma)^{2}} d u\right) .\right. \\
& \left.\cdot\left(\frac{\sigma_{1}-\beta}{\left(\sigma_{1}-\beta\right)^{2}+(t-\gamma)^{2}}\right)\right\} \\
& =\frac{\pi J_{0}}{\log X}=O\left(\frac{\log T}{\log \log T}\right) .
\end{aligned}
$$

This proves the Lemma. 


\section{Proof of Theorem 1}

From the results of Section 4 Theorem 1 follows from the identity (valid for $\left.\alpha \leq \sigma \leq \sigma_{1}=\alpha+(\log X)^{-1}\right)$

$$
\log F(\sigma+i t)=\log F\left(\sigma_{1}+i t\right)-\left(\sigma_{1}-\sigma\right) \frac{F^{\prime}}{F}\left(\sigma_{1}+i t\right)+\int_{\sigma}^{\sigma_{1}} K(u) d u
$$

where

$$
K(u) \equiv \frac{F^{\prime}}{F}\left(\sigma_{1}+i t\right)-\frac{F^{\prime}}{F}(u+i t)
$$

just as in [1].

\section{APPENDIX}

1. We can in this paper replace $F(s)$ by any function

$$
\sum_{n=1}^{\infty} \frac{a_{n}}{\lambda_{n}^{s}}
$$

where $1=\lambda_{1}<\lambda_{2}<\cdots$ is any increasing sequence of real numbers and $\left\{a_{n}\right\}$ with $a_{1}=1$ is any sequence of complex numbers such that the series

$$
\sum_{n=1}^{\infty} \frac{a_{n}}{\lambda_{n}^{s}}
$$

is absolutely convergent at some point of the complex plane. The condition $\alpha \geq \frac{1}{2}$ is unimportant. Any $\alpha$ will do. The only change is in place of Lemma 3 we have (by fixing $u$ to be large instead of $u=2) I=O\left(X^{2 u}(\log X)^{-1}\right)$ since $\left(F^{\prime} / F\right)(s+w)$ can be proved to be $O(1)$ for all $u$ exceeding some suitable $u_{0}$. We can now choose $X=(\log T)^{\lambda}$, where $\lambda$ is a sufficiently small positive constant. The rest of the proof is unaltered.

2. In our paper [2], in the condition $\alpha \geq \frac{1}{2}, \frac{1}{2}$ does not play any serious role, and the condition can be relaxed to any $\alpha \leq 1-\delta$.

3. In [2], we can (instead of the Euler product) work with the condition $F(1+i t)=O\left((\log T)^{A}\right)$ for $T-H \leq t \leq T+H$ where

$$
H=C(\log \log T)(\log \log \log T) .
$$

For, it follows that in $(\sigma \geq 1, T-3 H / 4 \leq t \leq T+3 H / 4)$ we have $\operatorname{Re} \log F(s) \leq$ $C \log \log T$ (not the same $C$ at all places) and by the Borel-Carathéodory theorem we can prove that in $\left(\sigma \geq 1+1 /(\log \log T), T-\frac{1}{2} H \leq t \leq T+\frac{1}{2} H\right)$ there holds $\log F(s)=O\left((\log \log T)^{2}\right)$. Now we can apply convexity arguments to obtain a bound for $|\log F(s)|$ in $\left(\sigma \geq \alpha+C^{\prime} / \log \log T, T-H / 3 \leq t \leq T+H / 3\right)$, not very different from the resuits proved in paper [2]. 
4. Without stating the most general results obtainable by the results of this paper, we can state results like this for example. Let

$$
F(s)=\zeta(s)+\sum_{n=1}^{\infty} \frac{a_{n}}{n^{s}}
$$

where

$$
\sum_{n \leq x} a_{n}=O\left(x^{(1 / 2)-\delta}\right)
$$

where $\delta>0$ is a constant. Let $T \geq T_{0}$. Then $F(s)$ has $\geq T^{1-\varepsilon}$ zeros in $\left(\sigma \geq \frac{1}{2}-C^{\prime \prime} / \log \log T, T \leq t \leq 2 T\right)$ where $C^{\prime \prime}$ depends only on $\varepsilon$ and other constant like $\delta$. The same is also true of the function $F(s)$ defined in $\sigma>0$ by

$$
\sum_{n=1}^{\infty} \frac{(-1)^{n-1}}{\lambda_{n}^{s}}
$$

where $1=\lambda_{1}\left\langle\lambda_{2}<\lambda_{3}<\cdots\right.$ and $\lambda_{n+1}-\lambda_{n}$ is both $\gg$ and $\ll 1$. (It may be noted that both $a_{n}$ and $\lambda_{n}$ can depend on $T$ ).

\section{References}

[1] Ramachandra, K., and A. Sankaranarayanan: On some theorems of Littlewood and Selberg I. - To appear.

[2] Ramachandra, K., and A. Sankaranarayanan: On some theorems of Littlewood and Selberg II. - Ann. Acad. Sci. Fenn. Ser. A I Math. 16, 1991, 131-137.

[3] Ramachandra, K.: Some problems of analytic number theory I. - Acta Arith. 31, 1976, 313-324.

K. Ramachandra

Tata Institute of Fundamental Research

School of Mathematics

Homi Bhabha Road

Bombay 400005

India

Received 6 June 1990
A. Sankaranarayanan Tata Institute of Fundamental Research School of Mathematics Homi Bhabha Road Bombay 400005 India 